\title{
Psychopharmaka und Psychotherapie - eine überwundene Dichotomie
}

Es hat eine lange Tradition, die Behandlung mit Psychopharmaka der mit Psychotherapie als unvereinbar gegenüber zu stellen. Zum einen wurde diskutiert, welche Behandlungsalternative die bessere therapeutische Wirkung habe, wobei implizit dann postuliert wurde, dass der Nachweis der therapeutischen Überlegenheit einer Methode automatisch den Ausschluss der anderen zur Konsequenz haben müsse. Zum anderen wurde aus grundsätzlichen theoretischen Überlegungen die Unvereinbarkeit der gleichzeitigen Anwendung von Psychopharmaka und Psychotherapie zum Axiom erhoben. Es ist ein großer theoretischer und praktischer Fortschritt, zu dem insbesondere die Verhaltenstherapie beigetragen hat, dass diese Dichotomie heute als überwunden gelten kann. Statt dessen wird eine sehr viel sachlichere und vor allem empirisch orientierte Diskussion geführt. Heute gilt der Grundsatz, dass ein Patient ein Anrecht auf diejenige Behandlung hat, die die bestmögliche Chance einer Besserung bietet. Aus klinischen, juristischen und ethischen Gründen ist jeder Therapeut verpflichtet, im konkreten Einzelfall zu prüfen, welche Behandlungsoption die beste Prognose verspricht. Das bedeutet, dass der Pharmakotherapeut den Patienten auch über psychotherapeutische Behandlungsmöglichkeiten aufklären muss, ebenso wie der Psychotherapeut sich einer unterlassenen Aufklärung schuldig machen kann, wenn er nicht auch auf pharmakotherapeutische Behandlungsmöglichkeiten oder gar -notwendigkeiten hinweist. Dies gilt für Therapiealternativen ebenso wie für Therapiekombinationen.

Mit dieser vorurteilsfreien Herangehensweise stellen sich bezüglich der gleichzeitigen Anwendung von Pharmakotherapie und Psychotherapie gerade aus verhaltenstherapeutischer Sicht eine Reihe von interessanten neuen Fragen, die über die vergleichende Wirksamkeitsdiskussion weit hinausreichen. Die aktuelle Diskussion um Psychopharmaka ist von dem Konzept getragen, dass ein bestimmtes Psychopharmakon für die Behandlung einer bestimmten Erkrankung einzusetzen ist - d.h. Antidepressiva für Depressionen oder Neuroleptika für schizophrene Erkrankungen. Dieses Konzept ist jedoch primär durch juristische Regularien bei der Zulassung von Arzneimitteln begründet, da die Zulassungsbehörden derzeit Studien verlangen, in denen definierte Erkrankungen das Behandlungsziel dargestellt haben. Diese Vorstellung ist jedoch wissenschaftlich nur bedingt begründbar. Statt dessen gilt seit den Anfängen der Psychopharmakotherapie das sogenannte Zielsyndromkonzept nach Freyhan. Damit ist gemeint, dass Psychopharmaka bestimmte Symptome bessern, unabhängig von ihrer nosologischen Einordnung. Antidepressiva können die Stimmung aufhellen und den Antrieb verbessern - auch dann, wenn der Patient beispielsweise unter einer schizophrenen Erkrankung oder hirnorganischen Störung und nicht unter einer Depression leidet. Neuroleptika werden eingesetzt zur Behandlung produktiver Symptomatik nicht nur bei schizophrenen Erkrankungen sondern ebenso im Delir oder bei wahnhaften Störungen beim Morbus Alzheimer.

Die moderne Pharmakologie versucht unter dem Stichwort der «Denosologisierung» dieses Zielsyndromkonzept noch weiter zu differenzieren. Alle bekannten Psychopharmaka binden gleichzeitig an sehr unterschiedliche Rezeptoren im zentralen Nervensystem. Jede dieser Rezeptoraffinitäten ist mit bestimmten Nebenwirkungen und auch Hauptwirkungen in Zusammenhang zu bringen. Dies zeigt sich nicht zuletzt in der Verhaltenspharmakologie darin, dass diese Substanzen in der Lage sind, Verhaltensänderungen auf sehr unterschiedlichen Ebenen zu bewirken. Es fehlt allerdings noch eine brauchbare wissenschaftliche Klassifikation solcher Einzelwirkdimensionen. So haben Psychopharmaka aller Substanzklassen neben antipsychotischen und antidepressiven oder sedierenden Wirkungen abhängig vom Rezeptor-Affinitätsprofil auch Effekte wie beispielsweise das selektive Unterdrücken von Vermeidungsverhalten bei gleichzeitigem Fortbestehen von Fluchtverhalten, die Förderung von Neugier und Explorationsverhalten, Stress distanzierende Wirkungen, vegetativ stabilisierende Wirkungen, Veränderungen von Dominanzund Konfliktverhalten, Besserung der Impulskontrolle usw. Die Einzelwirkungsdimensionen sind Krankheitsklassenübergreifend von Bedeutung und eröffnen aus verhaltenstherapeutischer Sicht interessante therapeutische Einsatzmöglichkeiten. Nimmt man diese Erkenntnisse zur Pharmakodynamik und Verhaltenspharmakologie ernst, dann eröffnet sich ein weites Spektrum an pharmakologischen Wirkungen, die sehr gezielt in einen verhaltensanalytischen Modellrahmen eingefügt werden können. So könnte eine Verbesserung der Impulskontrolle gleichzeitig beispielsweise durch Gabe einer

\begin{tabular}{ll}
\hline KARGER & @ 2001 S. Karger GmbH, Freiburg \\
Fax +49 761 4520714 & Accessible online at: \\
$\begin{array}{l}\text { E-mail Information@Karger.de } \\
\text { www.karger.com }\end{array}$ & www.karger.com/journals/ver
\end{tabular}

Prof. Dr. M. Linden

Abt. Verhaltenstherapie und Psychosomatik,

Rehabilitationsklinik Seehof der BfA

Lichterfelder Allee 55

D-14513 Teltow/Berlin (Deutschland) 
serotonergen Substanz und durch verhaltenstherapeutische Selbstkontrollstrategien therapeutisch angegangen werden.

Eine derartige differenzierte Betrachtung des Wechselspiels von pharmakotherapeutischen und psychotherapeutischen Interventionen bedarf allerdings noch einer umfassenden wissenschaftlichen Bearbeitung.

Die Pharmakopsychologie, die sich wissenschaftlich mit sol- chen Fragen befasst, ist zwar eine Disziplin mit einer langen Tradition, sie führt jedoch eher ein Schattendasein. Es wäre zu hoffen, dass dieser Wissenschaftszweig sowohl von den jeweiligen Fachvertretern wie vor allem auch von den Arzneimittelherstellern in der Zukunft mehr Aufmerksamkeit und auch materielle Unterstützung erhalten würde.

M. Linden, Teltow/Berlin 